\title{
Ultrafiltration rate is an important determinant of microcirculatory alterations during chronic renal replacement therapy
}

\author{
Gerke Veenstra ${ }^{1,34^{*}}$ (D), Andrius Pranskunas², Inga Skarupskiene², Vidas Pilvinis², Marc H. Hemmelder ${ }^{1}$, Can Ince In $^{3,4}$
} and E. Christiaan Boerma'

\begin{abstract}
Background: Hemodialysis (HD) with ultrafiltration (UF) in chronic renal replacement therapy is associated with hemodynamic instability, morbidity and mortality. Sublingual Sidestream Dark Field (SDF) imaging during HD revealed reductions in microcirculatory blood flow (MFI). This study aims to determine underlying mechanisms.

Methods: The study was performed in the Medical Centre Leeuwarden and the Lithuanian University of Health Sciences. Patients underwent 4-h HD session with linear UF. Nine patients were subject to combinations of HD and UF: $4 \mathrm{~h}$ of HD followed by $1 \mathrm{~h}$ isolated UF and $4 \mathrm{~h} \mathrm{HD}$ with blood-volume-monitoring based UF. Primary endpoint: difference in MFI before and after intervention. During all sessions monitoring included blood pressure, heartrate and SDF-imaging. Trial registration number: NCT01396980.

Results: Baseline characteristics were not different between the two centres as within the HD/UF modalities. MFI was not different before and after HD with UF. Total UF did not differ between modalities. Median MFI decreased significantly during isolated UF [2.8 (2.5-2.9) to $2.5(2.2-2.8), p=0.03]$. Baseline MFI of each UF session was correlated with MFI after the intervention $\left(r_{\mathrm{s}}=0.52, p=0.006\right)$.

Conclusion: During HD with UF or isolated HD we observed no changes in MFI. This indicates that non-flow mediated mechanisms are of unimportance. During isolated UF we observed a reduction in MFI in conjunction with a negative intravascular fluid balance. The correlation between MFI before and after intervention suggests that volume status at baseline is a factor in microvascular alterations. In conclusion we observed a significant decrease of sublingual MFI, related to UF rate during chronic renal replacement therapy.
\end{abstract}

Keyword: Microcirculation, Hemodialysis, Microvascular alterations, Ultrafiltration rate, Negative fluid balance

\section{Background}

Intermittent hemodialysis (HD) with concomitant ultrafiltration (UF) in chronic renal replacement therapy is associated with hemodynamic instability, usually referred to as intradialytic hypotension'. The incidence of this phenomenon ranges between 30 and 90\% depending on the definition on clinically relevant intradialytic hypotension. This unfavourable condition is not only associated with the inability to

\footnotetext{
* Correspondence: gerkevjr@zonnet.nl

'Medical Center Leeuwarden, P.O. Box 888, Leeuwarden 8934 AD, The Netherlands

${ }^{3}$ Translational Physiology, Academic Medical Center, Amsterdam, The Netherlands

Full list of author information is available at the end of the article
}

extract fluids adequately, but also with increased all-cause mortality, hospitalization for heart failure/volume overload and major adverse cardiac events [1-4]. In addition, intradialytic hypotension is likely to represent the tip of the iceberg with respect to consequences of changes in organ perfusion during HD. A striking discordance between hemodialysis-related symptoms or changes in (relative) blood volume and intradialytic hypotension has been reported $[5,6]$. Intradialytic hypotension is more likely to represent a late symptom of a pre-existing gradual reduction in blood flow during $\mathrm{HD}$, compensated by an increase in vascular resistance and cardiac performance. However, preexistent cardiac morbidity and concomitant treatment is 
likely to disturb this compensation mechanisms. More importantly, decreased left ventricular compliance as a result of increased heart mass and a rapidly descending systemic vascular resistance are both risk factors for a decreased cardiac output and potentially hypotension in dialysis patients [7]. Apart from non-circulatory effects of HD this discordance also represents a fundamental problem within the current clinical assumption that blood pressure is directly related to organ perfusion. To overcome this problem direct visualisation and quantification of the sublingual microcirculation with a hand-held device has been suggested by Bemelmans and co-workers, as a noninvasive tool to trace 'organ' perfusion during HD [8]. Direct in-vivo microscopy of the sublingual area with sidestream dark field (SDF) imaging during HD revealed marked reductions in microcirculatory blood flow in the absence of intradialytic hypotension in the vast majority of patients. Despite the potential of these observations many questions remain to be answered. The incidence, aetiology and clinical relevance of the microvascular alterations remain to be elucidated. This study has 2 major objectives: 1. Are we able to reproduce previous observations in a comparable subset of patients; and 2. Are the observed microcirculatory alterations the result of UF, HD or a combination?

\section{Methods}

\section{Study design and setting}

Phase I consisted of a multi-centre prospective observational study conducted between October 2011 and December 2012. Participating centres were the Medical Centre Leeuwarden, a tertiary teaching hospital in the Netherlands and The Hospital of Lithuanian University of Health Sciences, an academic medical centre in Kaunas, Lithuania. Local ethical committees of both hospitals approved the study and written informed consent was obtained from every patient, according to applicable laws. In phase II (2012) of the study all patients included in the Netherlands were additionally subject to a single-centre prospective interventional study to compare different combinations of HD and UF rates. Study design was registered in advance at Clinicaltrials.gov (NCT01396980).

\section{Intervention}

In phase I all patients were subject to their routine 4-h HD session, using a standard bicarbonate dialysate on normal temperature. During this period linear UF was maintained at a constant rate, in order to achieve a quantitative ultrafiltrate target, based upon the registered ideal dry weight of the patient. Primary endpoint is the difference in MFI between baseline and post intervention.

In phase II patients from the Netherlands were subject to 2 additional combinations of HD and UF: $4 \mathrm{~h}$ of HD alone followed by $1 \mathrm{~h}$ isolated UF, and $4 \mathrm{~h}$ HD plus UF based on blood-volume-monitoring (BVM) (5008 hemodialysis machine ${ }^{\oplus}$, Fresenius Medical Care) (Fig. 1) [9]. Sessions were assigned to each patient in random order and performed on the same day of the week; every patient served as his/her own reference. Primary endpoint is the difference in MFI between the HD/UF modalities post intervention.

\section{Measurements}

During all sessions standard hemodynamic monitoring included blood pressure, heartrate and peripheral oxygen saturation using pulse oximetry. Sublingual in-vivo microscopy with sidestream dark field (SDF)-imaging, incorporated in a small hand-held camera, was performed in all patients at baseline and at the end of each session. For each timeframe 3 steady images of at least $10 \mathrm{~s}$ were obtained and recorded on digital videotape (SONY videowalkman GV-D 1000E ${ }^{\oplus}$, Sony, Tokyo, Japan). Subsequent analysis was performed off-line and in random order with AVA software (Microvision Medical, Amsterdam, the Netherlands) [10]. Quantification of parameters of red blood cell velocity and capillary density was performed in accordance with an international consensus paper [11]. In short, red blood cell velocity in small vessels $(<20 \mu \mathrm{m})$ is scored semiquantitatively for each quadrant between 0 (stand still) and 3 (continuous normal flow) [12]. The average score of $3 \times 4$ quadrants is expressed as microvascular flow index (MFI). Total vessel density (TVD), as a determinant of capillary density, is calculated as the surface area of small vessels per $\mathrm{mm}^{2}$. Percentage of perfused vessels (PPV) is expressed as the percentage of perfused capillaries (MFI 2 and 3) divided by the total number of

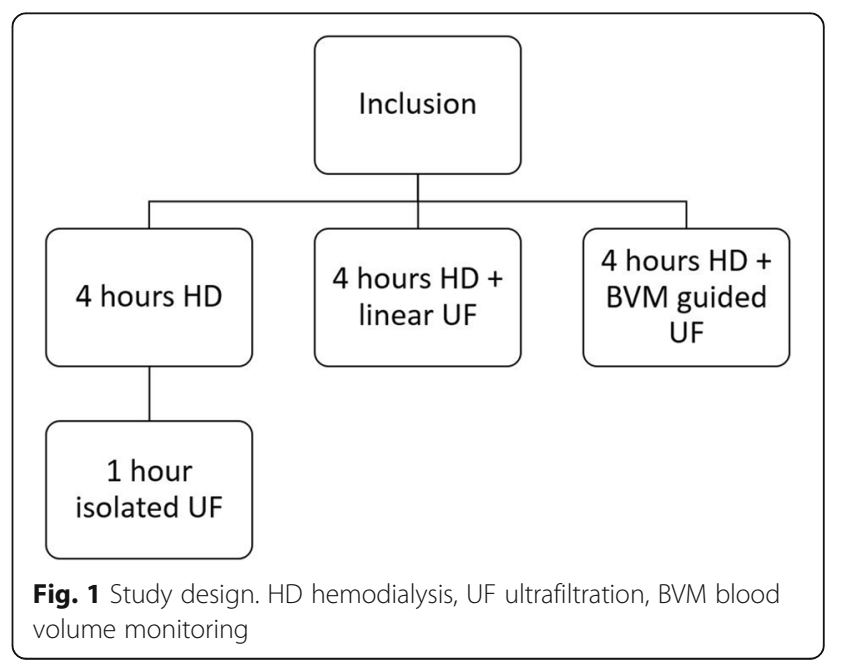


capillaries that crosses a grid of three horizontal and vertical equidistant lines. In phase II body composition monitoring (BCM; Fresenius Medical, Bad Homburg, Germany), based upon bioelectrical impedance analysis, and measurements of $\mathrm{N}$-terminal pro b-type natriuretic peptide (NT-proBNP) and Troponine T were additionally performed prior to and after each intervention $[13,14]$.

\section{Statistics}

All data are expressed as median [IQR]. Statistical analysis was performed with the Statistical Package for Social Sciences (SPSS 21, Chicago Illinois, USA). Due to the small sample size non-parametric tests for independent and paired data, as well as for correlation coefficients were applicable. A $p$-value $<0.05$ was considered statistically significant. Based upon previous publications a sample size of 8 patients for phase II was considered adequate to detect a difference of 0.7 arbitrary units in MFI between the different HD/UF modalities [8].

\section{Results}

\section{Phase I}

During a 15-months period in 2011-2012 28 patients were included in the study. Overall, baseline characteristics were not significantly different between the 2 centres (Table 1). The primary endpoint MFI was not different before and after $H D$ in combination with linear UF of 2.3 [1.3-3.2] l over a $4 \mathrm{~h}$ period (Table 2). In addition, hemodynamic variables did not change over time, with the exception of a small, but significant increase in peripheral oxygen saturation (Table 2).

\section{Phase II}

In a 2-month period in 2012 all 9 patients from the Netherlands that participated in phase I were included. There was no statistical difference between baseline characteristics of different HD/UF modalities (Table 3). Total UF did not differ between HD/UF modalities, with the exception of HD alone. However, UF rate was significantly higher in isolated UF $(p<0.001)$ in comparison to combined HD/UF modalities (Table 3). Median MFI decreased significantly during isolated UF [2.8 (2.5-2.9) to 2.5 (2.2-2.8), $p=0.03$ ], but remained unaltered during the other HD/UF modalities. We observed no significant difference between $\mathrm{HD}+$ linear UF and HD + BVMguide UF (Fig. 2). With the exception of isolated HD, BCM-derived overhydration and NT-pro-BNP decreased significantly during all $\mathrm{HD} / \mathrm{UF}$ modalities, indicating a similar trend in volume status.

Baseline MFI of each UF session (irrespective of UF modality) was significantly correlated with MFI after the intervention $\left(r_{\mathrm{s}}=0.52, p=0.006\right.$; Fig. 3a). The coefficient of correlation for pre- and post-intervention overhydration was also significant $\left(r_{\mathrm{s}}=0.75, p<0.001\right.$; Fig. $\left.3 \mathrm{~b}\right)$.

Table 1 Baseline characteristics phase I and II

\begin{tabular}{|c|c|c|c|c|}
\hline Variables & All $(n=28)$ & $\operatorname{LT}(n=19)$ & $\mathrm{NL}(n=9)$ & $p$-value \\
\hline Men, \% & 57 & 42 & 89 & 0.04 \\
\hline Age, years & 64 [53-74] & 60 [49-70] & 69 [55-78] & 0.29 \\
\hline Years on HD & $3[1-6]$ & $3[1-7]$ & $3[1-5]$ & 0.94 \\
\hline Remaining diuresis, l/24 h & $0.2[0-0.5]$ & $0.3[0-0.6]$ & $0.2[0-0.4]$ & 0.60 \\
\hline Weight, kg & 78 [67-87] & $73[66-79]$ & 85 [88-92] & 0.03 \\
\hline $\mathrm{BMl}, \mathrm{kg} / \mathrm{m} 2$ & 26 [24-28] & 27 [23-30] & 26 [25-28] & 0.94 \\
\hline UF volume, I & $2.3[1.3-3.2]$ & $2.6[1.6-3.3]$ & $1.7[1.2-2.1]$ & 0.10 \\
\hline \multicolumn{5}{|l|}{ Cause of ESRD, \% } \\
\hline Diabetes & 32 & 21 & 56 & \\
\hline Hypertension & 18 & 26 & 0 & \\
\hline ADPKD & 7 & 5 & 11 & 0.27 \\
\hline ATN & 14 & 16 & 11 & \\
\hline Miscellaneous & 29 & 32 & 22 & \\
\hline \multicolumn{5}{|l|}{ Drugs, \% } \\
\hline ß-blocker & 61 & 42 & 67 & 1.0 \\
\hline ACE inhibitor & 61 & 74 & 33 & 0.1 \\
\hline Calcium antagonist & 47 & 58 & 22 & 0.09 \\
\hline
\end{tabular}

LT Lithuania, NL Netherlands, HD hemodialysis, BMI body mass index, UF ultrafiltration, ESRD end stage renal disease, $A D K P D$ autosomal dominant polycystic kidney disease, ATN acute tubular necrosis, ACE angiotensin converting enzyme 
Table 2 Results phase I $(n=28)$. Hemodynamic and microcirculatory variables of small vessels ( $<20 \mu \mathrm{m})$ before and after hemodialysis in combination with linear ultrafiltration

\begin{tabular}{llll}
\hline Variables & Baseline & Post HD/UF & $p$-value \\
\hline Mean arterial pressure, $\mathrm{mmHg}$ & $93[76-111]$ & $96[84-110]$ & 0.39 \\
Heartrate, beats/min & $69[62-80]$ & $73[60-84]$ & 0.24 \\
$\mathrm{SpO}_{2}, \%$ & $97[96-98]$ & $98[98-99]$ & 0.009 \\
$\mathrm{MFI}, \mathrm{AU}$ & $3[2.8-3]$ & $3[2.8-3]$ & 0.55 \\
$\mathrm{TVD}, \mathrm{mm} / \mathrm{mm}^{2}$ & $22.2[18-29.8]$ & $22.7[19.9-29]$ & 0.11 \\
$\mathrm{PPV}, \%$ & $98[96-100]$ & $98[96-99]$ & 0.35 \\
\hline
\end{tabular}

$\mathrm{HD}$ hemodialysis, UF ultrafiltration, $\mathrm{SpO}_{2}$ peripheral oxygen saturation, $\mathrm{MFI}$ microvascular flow index, $A U$ arbitrary units, TVD total vessel density, PVD perfused vessel density, PPV percentage of perfused vessel

\section{Discussion}

In phase I of the study we did not observe a reduction in sublingual microvascular blood flow or capillary density during HD in combination with linear UF. In an attempt to unravel the aetiology of previously observed alterations in microvascular blood flow during combined HD/UF we changed the ultrafiltration modalities in a subset of patients in phase II. During isolated HD we observed no changes in microvascular blood flow. This indicates that potential non-flow mediated mechanisms for microvascular derangements, such as hemodialysis-induced inflammation and hypercoagulation, are unlikely to play an important role $[15,16]$. However, during isolated UF, a modality with the highest UF rate in which the influence of $\mathrm{HD}$ itself is absent, we were indeed able to reproduce the reduction in microvascular blood flow, as observed by others $[8,17]$. This suggests that the observed reduction in microvascular blood flow may be the result of a negative intravascular fluid balance. In case the UF rate exceeds the ability to mobilize interstitial fluids towards the intravascular space, an increase in vascular resistance or viscosity causes a reduction in microvascular blood flow. The fact that Bemelmans et al. observed a marked attenuation of impaired microvascular blood flow during autotransfusion with a Trendelenburg manoeuvre after HD/UF is also in line with this aetiology [8].

An important issue is the question why we did not observe previously reported microcirculatory alterations during HD in combination with linear UF. At first glance there are important similarities with both articles: UF was $2.5[1.6-3.5] 1$ and $2.5 \pm 0.881$ respectively, and over a similar period of time $[8,17]$. But a closer look reveals a marked reduction in MFI at baseline in both studies; 2.8 [2.5-5] and $2.7 \pm 0.5$ versus 3 [2.8-3] in our study. Baseline values of these previous publications indicate pre-existing microvascular derangement prior to the start of $\mathrm{HD} / \mathrm{UF}$, since they are outside the range of healthy volunteers $[18,19]$. A secondary analysis of our data in
Table 3 Results phase II $(n=9)$. Laboratory data, microcirculatory variables of small vessels ( $<20 \mu \mathrm{m})$ and bioelectrical impedance analysis before and after intervention

\begin{tabular}{|c|c|c|c|}
\hline Variables & Baseline & Post-intervention & $p$-value \\
\hline \multicolumn{4}{|l|}{ UF isolated } \\
\hline UF & - & $1.7[1.2-2]$ & \\
\hline UF rate, I/h & - & $1.7[1.2-2]^{\dagger}$ & \\
\hline $\mathrm{MFI}, \mathrm{AU}$ & $2.8[2.5-2.9]$ & $2.5[2.2-2.8]$ & 0.03 \\
\hline $\mathrm{TVD}, \mathrm{mm} / \mathrm{mm}^{2}$ & $17.7[16.5-18.4]$ & 18.7 [16.1-20.3] & 0.26 \\
\hline Hematocrit, \% & 37 [35-39] & 39 [36-44] & 0.11 \\
\hline NT-pro-BNP, pmol/l & 599 [215-1702] & 580 [158-1440] & 0.01 \\
\hline Troponine T, ng/l & 90 [60-150] & 87 [56-130] & 0.08 \\
\hline BCM overhydration, I & $1.4[0.6-3.2]$ & $0.3[-0.1-0.8]$ & 0.02 \\
\hline \multicolumn{4}{|l|}{ HD isolated } \\
\hline UF & - & $0[0-0]$ & \\
\hline UF rate, $1 / h$ & - & $0[0-0]^{\dagger}$ & \\
\hline $\mathrm{MFI}, \mathrm{AU}$ & $2.8[2.5-2.9]$ & $2.8[2.7-2.9]$ & 0.61 \\
\hline TVD, mm/mm ${ }^{2}$ & $17.7[16.5-18.4]$ & 18.6 [14.6-19.7] & 0.86 \\
\hline Hematocrit, \% & 37 [35-39] & NA & \\
\hline NT-pro-BNP, pmol/l & 599 [215-1702] & NA & \\
\hline Troponine T, ng/l & 90 [60-150] & NA & \\
\hline BCM overhydration, I & $1.4[0.6-3.2]$ & NA & \\
\hline
\end{tabular}

$\mathrm{HD}+$ linear UF

UF

$1.7[1.2-2.1]$

UF rate, $l / h$

$0.42[0.3-5.1]^{\dagger}$

$\mathrm{MFI}, \mathrm{AU}$

$2.9[2.5-3]$

$2.6[2.2-2.9]$

0.12

TVD, $\mathrm{mm} / \mathrm{mm}^{2}$

17.8 [16.6-18.8]

19.8 [17.9-21.5]

0.07

Hematocrit, \%

T-pro-BNP, pmol//

38 [35-40]

40 [35-41]

0.18

Troponine T, ng/l

618 [279-1926]

536 [167-1003]

0.01

BCM overhydration, I

94 [61-179]

84 [62-139]

0.02

HD + BVM-guided UF

UF

UF rate, $I / h$

$\mathrm{MFI}, \mathrm{AU}$

TVD, $\mathrm{mm} / \mathrm{mm}^{2}$

$1.8[0.5-5.3]$

$0.3[-0.9-3.2]$

0.02

Hematocrit, \%

NT-pro-BNP, pmol/I

Troponine T, ng/l

BCM overhydration, I

2.0 [1.5-2.1]

$0.5[0.39-0.52]^{\dagger}$

2.8 [1.9-2.9]

0.06

$2.8[2.5-3]$

18.2 [16.9-20.6]

0.77

37 [34-39] 39 [35-42]

0.12

574 [229-2011] 511 [172-1163]

0.01

ultrafiltration, $H D$ hemodialysis, $B V M$ blood volume monitoring, $M F$ microvascular flow index, TVD total vessel density, PPV percentage of perfused vessels, NT-pro-BNP n-terminal pro b-type natriuretic peptide, BCM body composition monitoring ${ }^{\dagger} p<0.001$ across different HD/UF modalities

phase II revealed a significant correlation between MFI at baseline and MFI after the intervention, indicating that indeed volume status at baseline is an additional factor in 


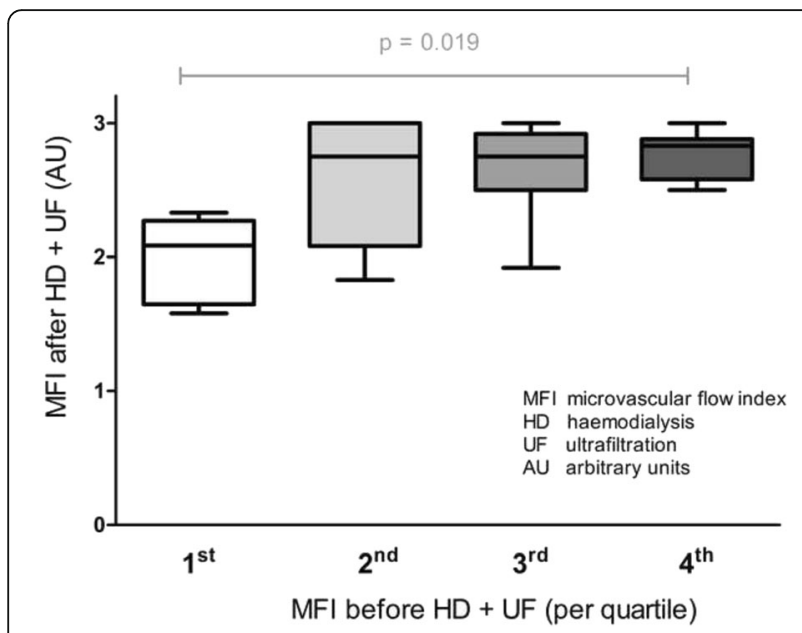

Fig. 2 Distribution of post-intervention microvascular alterations per quartile of pre-intervention microvascular blood flow. P-value across groups

the development of microvascular alterations during UF, irrespective of its modality. This suggest that our patients were less prone for microcirculatory changes, but that a higher rate of isolated ultrafiltration can result in impaired microperfusion in this group. Furthermore, our data suggest that $56 \%$ of the postintervention overhydration is caused by pre-treatment overhydration (Fig. 3b).

Further studies are needed to investigate a potential correlation between ultrafiltration-derived changes in microvascular flow and morbidity and/or mortality in hemodialysis patients.

The limitations of the study are related to the small sample size in phase II. We anticipated a potential difference in MFI between the UF modalities, based upon previous observations. However, the observed changes in MFI were considerably smaller. As a consequence we may have been unable to detect an existing difference in sublingual microvascular blood flow between HD + linear UF and HD + BVM-guided UF (type I error).

\section{Conclusions}

In conclusion we observed a significant decrease of sublingual microvascular blood flow due to rapid isolated ultrafiltration. Additional interventions with different combinations of HD and UF revealed that HD per se is not associated with changes in microvascular flow. During ultrafiltration over a longer period of time, and in combination with hemodialysis, baseline abnormalities were associated abnormal microvascular blood flow at the end of the renal replacement session. By design this

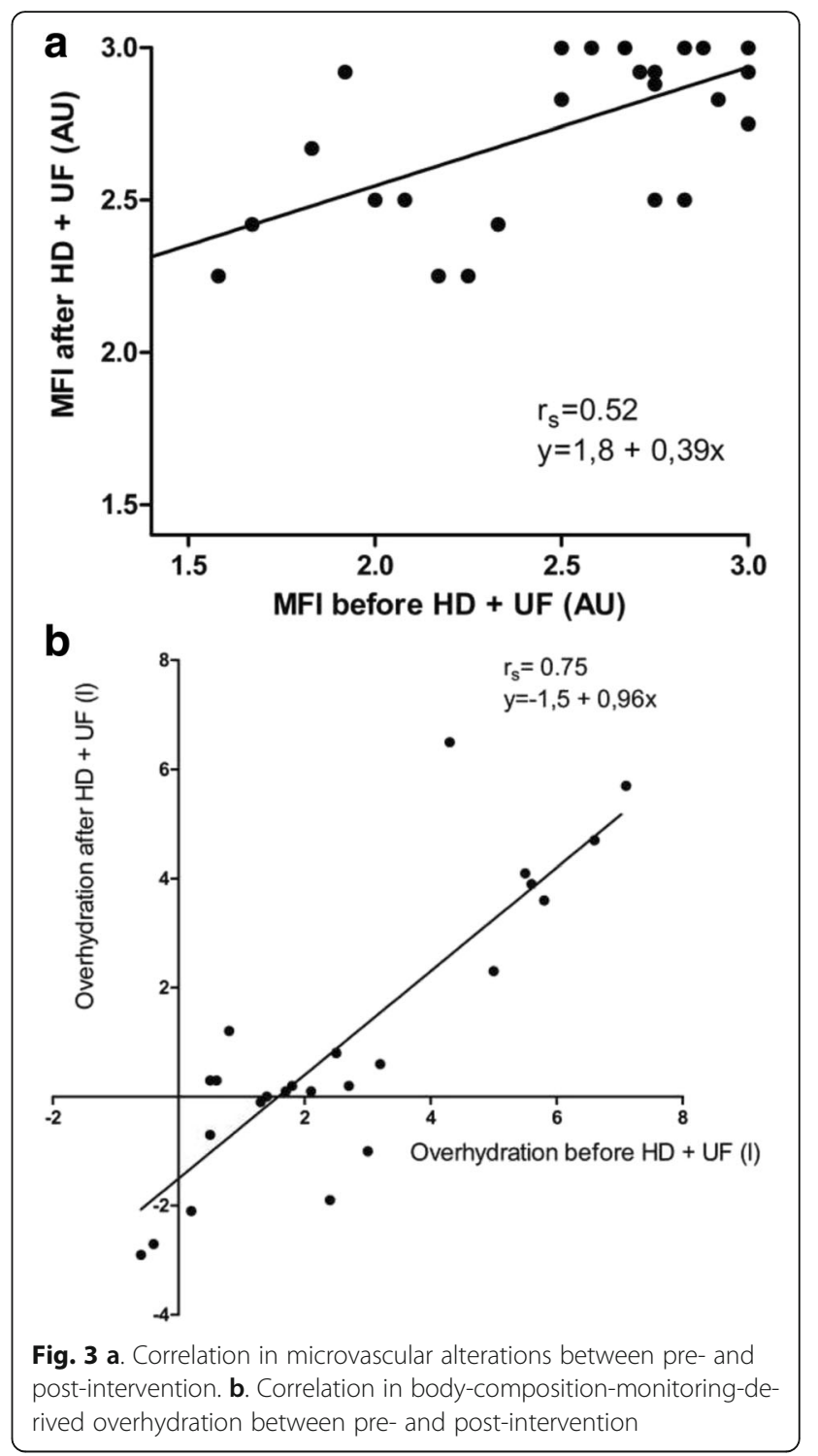

study is not suitable to establish the clinical relevance of the observed microvascular alterations.

\section{Abbreviations}

BVM: Blood-volume-monitoring; HD: Hemodialysis; MFl: Microcirculatory blood flow; NT-proBNP: N-terminal pro b-type natriuretic peptide; PPV: Percentage of perfused vessels; SDF: Sublingual Sidestream Dark Field; Trop-T: Troponine T; TVD: Total vessel density; UF: Ultrafiltration

\section{Acknowledgements}

Not applicable.

\section{Funding}

No funding was used for this study.

\section{Availability of data and materials}

Because of patient confidentiality data is not available.

\section{Authors' contributions}

Study design: GV, AP, IS, VP, MH, CI, EB. Data analysis and interpretation: GV, $A P$, EB. Drafting of the manuscript: GV, EB. Statistical analysis: GV, EB. All authors read and approved the final manuscript. 


\section{Competing interests}

$\mathrm{GV}, \mathrm{AP}, \mathrm{IS}, \mathrm{VP}, \mathrm{MH}$ and $\mathrm{EB}$ have no conflicts of interest. $\mathrm{Cl}$ is the inventor of SDF technology, which is commercialized by MicroVision Medical. He has been a consultant for this company in the past, but he has broken all contact with this company for more than 2 years now, and he has no competing interests other than his commitment to promote the importance of the microcirculation in the care of patients.

\section{Consent for publication}

Not applicable.

\section{Ethics approval and consent to participate}

Local ethical committees (Regionale Toetsingscommissie Patiëntgebonden Onderzoek Leeuwarden and Kauno Regioninis Biomedicininiu Tyrimu Etikos Komitetas) of both hospitals approved the study and written informed consent was obtained from every patient, according to applicable laws.

\section{Author details}

${ }^{1}$ Medical Center Leeuwarden, P.O. Box 888, Leeuwarden 8934 AD, The Netherlands. ${ }^{2}$ Lithuanian University of Health Sciences, Kaunas, Lithuania. ${ }^{3}$ Translational Physiology, Academic Medical Center, Amsterdam, The Netherlands. ${ }^{4}$ Erasmus MC University Hospital Rotterdam, Rotterdam, The Netherlands.

Received: 13 September 2016 Accepted: 9 February 2017

Published online: 20 February 2017

\section{References}

1. Chao CT, Huang JW, Yen CJ. Intradialytic hypotension and cardiac remodeling: a vicious cycle. Biomed Res Int. 2015;2015:724147.

2. Mclntyre CW, Goldsmith DJ. Ischemic brain injury in hemodialysis patients: which is more dangerous, hypertension or intradialytic hypotension? Kidney Int. 2015;87(6):1109-15

3. Sands JJ, Usvyat LA, Sullivan T, Segal JH, Zabetakis P, Kotanko P, Maddux FW, Diaz-Buxo JA. Intradialytic hypotension: frequency, sources of variation and correlation with clinical outcome. Hemodial Int. 2014;18(2):415-22.

4. Flythe JE, Xue H, Lynch KE, Curhan GC, Brunelli SM. Association of mortality risk with various definitions of intradialytic hypotension. J Am Soc Nephrol. 2015;26(3):724-34

5. Meredith DJ, Pugh CW, Sutherland S, Tarassenko L, Birks J. The relationship between symptoms and blood pressure during maintenance hemodialysis. Hemodial Int. 2015;19(4):543-52.

6. Booth J, Pinney J, Davenport A. Do changes in relative blood volume monitoring correlate to hemodialysis-associated hypotension? Nephron Clin Pract. 2011;117(3):c179-83.

7. Straver B, Roggekamp MC, de Vries PM, ter Wee PM. Systemic vascular resistance in intradialytic hypotension determined by means of impedance cardiography. Blood Purif. 1998;16(5):281-9.

8. De Backer D, Ortiz JA, Salgado D. Coupling microcirculation to systemic hemodynamics. Curr Opin Crit Care. 2010;16(3):250-4

9. Weir MR. Relative plasma volume monitoring and blood pressure control: an overlooked opportunity to achieve dry weight in the hemodialysis patient. Hypertension. 2010;55(2):226-7.

10. Dobbe JGG, Streekstra GJ, Atasever B, van Zijderveld R, Ince C. Measurement of functional microcirculatory geometry and velocity distributions using automated image analysis. Med Biol Eng Comput. 2008;46(7):659-70.

11. Ince C. Go with the flow-recruit the microcirculation! Intensive Care Med. 2006:32(4):488-9.

12. Bartels SA, Bezemer R, Milstein DMJ, Radder M, Lima A, Cherpanath TGV, Heger $M$, Karemaker JM, Ince C. The microcirculatory response to compensated hypovolemia in a lower body negative pressure model. Microvasc Res 2011;82(3):374-80.

13. Chamney PW, Wabel P, Moissl UM, Müller MJ, Bosy-Westphal A, Korth O, Fuller NJ. A whole-body model to distinguish excess fluid from the hydration of major body tissues. Am J Clin Nutr. 2007:85(1):80-9.

14. Wabel P, Chamney P, Moiss U, Jirka T. Importance of whole-body bioimpedance spectroscopy for the management of fluid balance. Blood Purif. 2009;27(1):75-80.

15. Zimmermann J, Herrlinger S, Pruy A, Metzger T, Wanner C. Inflammation enhances cardiovascular risk and mortality in hemodialysis patients. Kidney Int. 1999:55(2):648-58.
16. Topçiu-Shufta V, Haxhibeqiri V, Begolli L, Baruti-Gafurri Z, Veseli S, Haxhibeqiri S, Miftari R, Kurti L, Avdiu D. Correlation of inflammation and lipoprotein (a) with hypercoagulability in hemodialysis patients. Med Arch. 2015;69(4):232-5.

17. Meinders AJ, Nieuwenhuis L, Ince C, Bos WJ, Elbers PW. Haemodialysis impairs the human microcirculation independent from macrohemodynamic parameters. Blood Purif. 2015;40(1):38-44.

18. Edul VSK, Enrico C, Laviolle B, Vazquez AR, Ince C, Dubin A. Quantitative assessment of the microcirculation in healthy volunteers and in patients with septic shock. Crit Care Med. 2012;40(5):1443-8.

19. Aykut G, Veenstra G, Scorcella C, Ince C, Boerma C. Cytocam-IDF (incident dark field illumination) imaging for bedside monitoring of the microcirculation. Intensive Care Med Exp. 2015;3(1):40.

\section{Submit your next manuscript to BioMed Central and we will help you at every step:}

- We accept pre-submission inquiries

- Our selector tool helps you to find the most relevant journal

- We provide round the clock customer support

- Convenient online submission

- Thorough peer review

- Inclusion in PubMed and all major indexing services

- Maximum visibility for your research

Submit your manuscript at www.biomedcentral.com/submit
Biomed Central 